PREPARED FOR THE U.S. DEPARTMENT OF ENERGY, UNDER CONTRACT DE-AC02-76CH03073

PPPL-3924

PPPL-3924

UC-70

Microwave Imaging Reflectometry

for the Measurement of Turbulent Fluctuations in Tokamaks

by

E. Mazzucato

February 2004

M

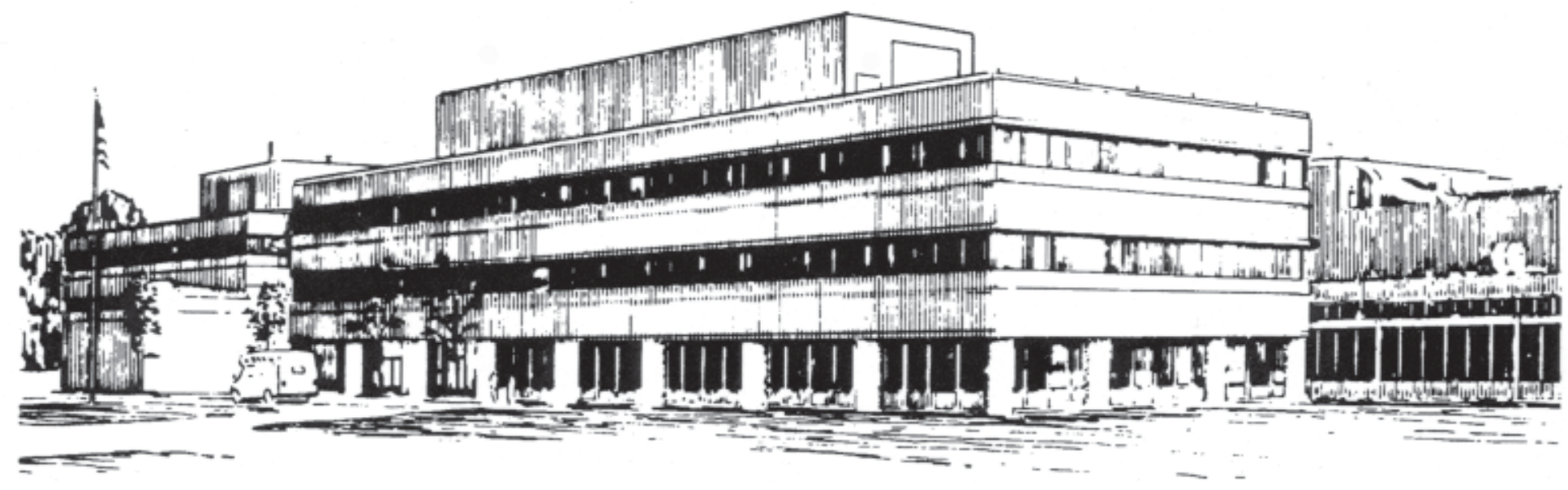

PRINCETON PLASMA PHYSICS LABORATORY PRINCETON UNIVERSITY, PRINCETON, NEW JERSEY 


\section{PPPL Reports Disclaimer}

This report was prepared as an account of work sponsored by an agency of the United States Government. Neither the United States Government nor any agency thereof, nor any of their employees, makes any warranty, express or implied, or assumes any legal liability or responsibility for the accuracy, completeness, or usefulness of any information, apparatus, product, or process disclosed, or represents that its use would not infringe privately owned rights. Reference herein to any specific commercial product, process, or service by trade name, trademark, manufacturer, or otherwise, does not necessarily constitute or imply its endorsement, recommendation, or favoring by the United States Government or any agency thereof. The views and opinions of authors expressed herein do not necessarily state or reflect those of the United States Government or any agency thereof.

\section{Availability}

This report is posted on the U.S. Department of Energy's Princeton Plasma Physics Laboratory Publications and Reports web site in Fiscal Year 2004. The home page for PPPL Reports and Publications is: http://www.pppl.gov/pub_report/

DOE and DOE Contractors can obtain copies of this report from:

U.S. Department of Energy

Office of Scientific and Technical Information

DOE Technical Information Services (DTIS)

P.O. Box 62

Oak Ridge, TN 37831

Telephone: (865) 576-8401

Fax: (865) 576-5728

Email: reports@adonis.osti.gov

This report is available to the general public from:

National Technical Information Service

U.S. Department of Commerce

5285 Port Royal Road

Springfield, VA 22161

Telephone: $1-800-553-6847$ or

(703) $605-6000$

Fax: (703) 321-8547

Internet: http://www.ntis.gov/ordering.htm 


\title{
Microwave imaging reflectometry for the measurement of turbulent fluctuations in tokamaks
}

\author{
E. Mazzucato ${ }^{\text {a) }}$ \\ Princeton Plasma Physics Laboratory, Princeton, New Jersey 08543
}

\begin{abstract}
This article describes a numerical study of microwave reflectometry for the measurement of turbulent fluctuations in tokamak-like plasmas with a cylindrical geometry. Similarly to what was found previously in planestratified plasmas, the results indicate that the characteristics of density fluctuations cannot be uniquely determined from the reflected waves if the latter are allowed to propagate freely to the point of detection, as in standard reflectometry. Again, we find that if the amplitude of fluctuations is below a threshold that is set by the spectrum of poloidal wave numbers, the local characteristics of density fluctuations can be obtained from the phase of reflected waves when these are collected with a wide aperture antenna, and an image of the cutoff is formed onto an array of phase-sensitive detectors.
\end{abstract}

Key words: Tokamak, anomalous transport, plasma turbulence, short-scale density fluctuations, microwave imaging reflectometry.

Subject classification: Te, D2, K0

a) Electronic mail: mazzucato@pppl.gov 


\section{INTRODUCTION}

Microwave reflectometry [1], a radar technique for the detection of fluctuations using the reflection of electromagnetic waves from a plasma cutoff, has found extensive use for the detection of short-scale turbulent fluctuations in tokamaks - the probable cause of anomalous transport in this type of magnetic configurations [2,3]. However, the extraction of any quantitative information from the measured signals is always very difficult and often impossible. This is caused by two phenomena. Firstly, the high sensitivity of reflectometry to plasma fluctuations makes its response non-linear, as demonstrated by the very first application of this technique to tokamaks [4]. The second reason, which is more subtle and was not promptly understood [5], is due to fact that when the plasma permittivity fluctuates perpendicularly to the direction of propagation of the probing beam, as in the case of tokamak plasmas where turbulent fluctuations vary in both radial and poloidal directions, the spectral components of the backward wave propagate in different directions. This may result in a complicated interference pattern on the detection plane, from which it is very difficult to extract any quantitative information on fluctuations under investigation.

This can be understood by considering a simple case where the wave permittivity has the form $\square=\square(r)+\square(r, \square)$ [where $\Pi \longrightarrow<1$ is the fluctuating component and $(r, \square)$ is a system of coordinates representing the radial and poloidal directions of a tokamak plasma], and by assuming that the reflected wave near the cutoff can be cast in the form $E=\exp (i \tilde{\square})$, with $\tilde{\square}(\square)$ given by the geometric optics approximation (phase screen model) $[5,6]$. Since the phase of the probing wave is the cumulative result of many random contributions, we may assume that $\tilde{\square}(\square)$ is a normal random variable with mean $<\tilde{\square}>=0$, variance $\square_{\square}^{2} \equiv<\tilde{\square}^{2}>$ and autocorrelation $\square(\square) \equiv<\tilde{\square}(\square) \tilde{\square}\left(\square+\square>/ \square_{\square}^{2}\right.$. From this, we get that the first moment of the wave amplitude (i.e., the amplitude of the 
coherent specular reflection) is $\left\langle E>=\exp \left(\square \square_{\square}^{2} / 2\right)\right.$, and thus it is a decreasing function of $\square_{\square}$. From the joint probability density [7]

$$
P\left(\tilde{\square}_{1}, \tilde{\square}_{2}\right)=\frac{1}{2 \square \square_{\square}^{2}\left(1 \square\left[\square_{\square}^{2}\right)^{1 / 2}\right.} \exp \underset{\square}{\square} \frac{\square_{1}^{2} \square 2 \square_{\square} \square_{1} \square_{2}+\square_{2}^{2}}{2 \square_{\square}^{2}\left(1 \square\left[\square_{\square}^{2}\right)\right.} \stackrel{E}{E},
$$

where $\tilde{\square}_{1} \equiv \tilde{\square}(\square)$ and $\tilde{\square}_{2} \equiv \tilde{\square}(\square+\square$, we get the second moment of the wave amplitude $<E_{1} E_{2}^{*}>=\exp \left[\square \square_{\square}^{2}(1 \square \measuredangle \zeta)\right]$, which shows that the signal correlation length is also a decreasing function of $\square_{\square}$. The normalized autocorrelation of $E$ is then given by

$$
\square_{E} \equiv \frac{<\tilde{E}_{1} \tilde{E}_{2}^{*}>}{<|\tilde{E}|^{2}>}=\frac{e^{\square_{\square}^{2} \square} \square 1}{e^{\square_{\square}^{2}} \sqcap 1} .
$$

From this, we get $\square_{E} \square \square_{\square}$ for $\square_{\square}^{2}<<1$. On the other hand, for $\square_{\square}^{2}>>1$, by taking $\square_{\square} \square \exp \left(\square \square^{2} / 2 \square_{\square}^{2}\right)$ and making the approximation $\square \square 1 \square \square^{2} / 2 \square_{\square}^{2}$ for $\square^{2} \ll \square_{\square}^{2}$, we obtain $\square_{E} \square \exp \left(\square^{2} \square_{\square}^{2} / 2 \square_{\square}^{2}\right)$. To summarize, when $\square_{\square}^{2}>>1$, the correlation length of the wave amplitude is a factor of $\square_{\square}$ smaller than the correlation length of $\tilde{\square}$.

In conclusion, $\square_{\square}$ cannot be derived from the measured value of $\square_{E}$ without knowledge $\square_{\square}$. On the other hand, the latter cannot be measured when the reflected waves are allowed to propagate freely to the detection plane where they may form a chaotic interference pattern. In a series of papers [8-10], the author has discussed the possibility of overcoming this difficulty by collecting the reflected waves with a wide aperture optical system forming an image of the cutoff onto an array of phase sensitive detectors. This should allow a measurement of the autocorrelation function Lb$_{\square}$, and hence information on the structure of plasma density fluctuations near the cutoff. Such a reflectometry scheme - named Microwave Imaging Reflectometry - was the result of an extensive series of numerical simulations where, as in the previous paragraph, a planestratified plasma equilibrium was used in conjunction with a field of two dimensional fluctuations. In this paper, we reanalyze the same problem using a plasma equilibrium 
configuration with a more realistic cylindrical geometry.

\section{PHYSICAL MODEL}

As in previous work [8-10], for the sake of simplicity we will consider only plasmas with sufficiently low magnetic shear to make the coupling between the two modes of wave propagation negligible. Extension to the case of large magnetic shears is considerably more cumbersome, but straightforward.

We assume that the density $(n)$ of a cylindrically symmetric plasma is perturbed by a field of 2D density fluctuations $(\tilde{n})$ with the spatial distribution

$$
\frac{\tilde{n}(r, \square)}{n(r)}=\underset{p=1}{\square} \underset{q=1}{\square} \square_{p q} \cos \left(p \square r+\square_{p q}\right) \cos (q \square),
$$

where $(r, \square)$ are cylindrical coordinates. This spectrum of fluctuations consists of $P \times Q$ discrete components with radial wave number $k_{r}=p \square(/ / \equiv$ constant $)$, poloidal number $q$, random phase $\square_{p q}$ and amplitudes $\square_{p q}$. Similarly to Ref. [8], for the latter we take the distribution

$$
\square_{p q}^{2} \quad p \exp \left[\square\left(p \square \square \square_{r}\right)^{2} \square\left(q / q_{0}\right)^{2}\right]
$$

where the constants $\square_{r}=\square P / 2$ and $q_{0}$ represent the spectral width of fluctuations in the radial and poloidal directions, respectively. At the cutoff, the poloidal spectral width can also be expressed in terms of the wave number $\square_{p}=q_{0} / r_{c}$. Throughout this article we will use $P=21$ and $Q=101$.

The probing wave is launched in the radial direction from $r=r_{0}$ with the Gaussian amplitude profile

$$
E_{0}(\square)=e^{\square\left(\square / \square_{0}\right)^{2}}
$$

where $\square_{0}$ is a constant. Since we assume $\square_{0}<<$, Eq. (5) can also be cast in the form 


$$
E_{0}(\square) \square \frac{\square_{0}}{2 \sqrt{\square}} \underset{m=\square}{\square=+} e^{\square\left(m \square_{0} / 2\right)^{2}} e^{i m \square} \equiv \underset{m=\square}{\square=+} g_{m} e^{i m \square} .
$$

The total amplitude $(E)$ of the wave, that without loss of generality we assume propagating in the ordinary mode, is expressed as the sum of $2 N+1$ independent solutions of the wave equation

$$
E(r, \square)=\square_{n=\square N}^{N} c_{n} E_{n}(r, \square),
$$

where $N \gg Q$ (to be determined). The functions $E_{n}$ are cast in the form

$$
E_{n}(r, \square)=\underset{m=\square N}{\square} f_{m n}(r) e^{i m \square},
$$

where $f_{m n}(r)$ are solutions of the system of $2 N+1$ ordinary differential equations

$$
\begin{aligned}
& \frac{d^{2} f_{m n}}{d r^{2}}+\frac{1}{r} \frac{d f_{m n}}{d r}+k_{0}^{2}\left(\square \square \square_{m}^{2}\right) f_{m n}+k_{0}^{2}(\square \square 1) \\
& \times \underset{p=1}{P} \underset{q=1}{Q}\left[\frac{\square_{p q}}{2} \cos \left(p \square r+\square_{p q}\right)\left(f_{(m \square q) n}+f_{(m+q) n}\right)\right]=0 \\
& \quad(m=-N,-N+1 \ldots \ldots . . N),
\end{aligned}
$$

with $\square=1 \square\left(\square_{p} / \square\right)^{2}$ (the unperturbed permittivity), $\square_{p}=\left(4 \square n e^{2} / m_{e}\right)^{1 / 2}$ (the plasma frequency), $\square_{m}=m / k_{0} r$ and $k_{0}=\square / c$ (the probing wave number). These equations, which are derived by inserting Eqs. (3) and (8) into the wave equation and by performing a Fourier expansion in $\square$, can be solved with the method of Runge-Kutta.

The coefficients $c_{n}$ in Eq. (7) are obtained by imposing that the wave field at $r=r_{0}$ is the sum of the incoming probing wave (Eq. (6)) and an outgoing reflected wave

$$
E_{r}(\square)=\underset{m=\square N}{\square} a_{m} e^{i m \square} .
$$

From this we get a first set of $2 N+1$ equations 


$$
\square_{n=\square N}^{N} f_{m n}\left(r_{0}\right) c_{n} \square a_{m}=g_{m} \quad, \quad(m=-N,-N+1 \ldots \ldots . . N)
$$

Another set of equations can be derived from the expressions

$$
E_{f}(r, \square)=\square_{m=\square}^{m=} g_{m} \frac{H_{m}^{(2)}\left(k_{0} r\right)}{H_{m}^{(2)}\left(k_{0} r_{0}\right)} e^{i m \square}
$$

and

$$
E_{b}(r, \square)=\square_{m=\square N}^{N} a_{m} \frac{H_{m}^{(1)}\left(k_{0} r\right)}{H_{m}^{(1)}\left(k_{0} r_{0}\right)} e^{i m \square}
$$

representing the solutions of the wave equation that at $r=r_{0}$ coincide with Eqs. (6) and (10), respectively. In these expressions, $H_{m}^{(1)} \equiv J_{m}+i Y_{m}$ and $H_{m}^{(2)} \equiv J_{m} \square i Y_{m}$ are the Hankel functions [11] which satisfy the recurrence relation

$$
2 F_{m \downarrow}(z)=F_{m \square 1}(z) \square F_{m+1}(z) .
$$

From the $r$-derivatives, then, we obtain a second set of equations

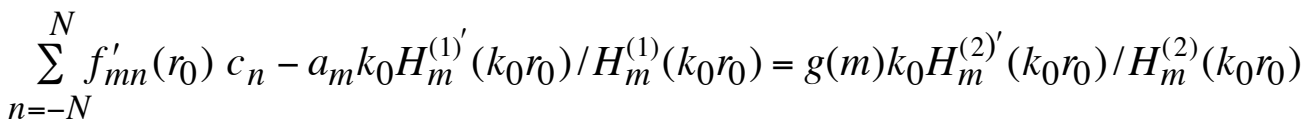

$$
\begin{aligned}
& (m=-N,-N+1 \ldots \ldots . . N),
\end{aligned}
$$

which together with Eq. (11) determine the values of $a_{n}$ and $c_{n}$.

In the following, Eq. (14) will be referred to as the backward field. Outside of the plasma region, $E_{b}$ coincides with the reflected wave, while inside the plasma region, it represents a virtual field that an observer in free space could measure by using an optical system to map the plasma region onto an array of detectors located at the image plane.

Finally, the integer $N$ must be chosen large enough to make the results significantly unchanged by any increase in its value. This condition, verified a posteriori, allows the closure of the system of equations by setting to zero all terms $f_{(m \pm q) n}$ with $|m \pm q|>N$. The numerical simulations of this paper have been performed using values of $N$ in the 
range $200 \leq N \leq 250$.

\section{NUMERICAL RESULTS}

The numerical results described in this section refer to the case of a cylindrical plasma with a radius of $r_{b}=80 \mathrm{~cm}$ and the density profile of Fig. 1 (derived from a typical plasma equilibrium of the Tokamak Fusion Test Reactor [5]). The probing wave has a frequency of $75 \mathrm{GHz}$ and is launched from $r_{0}=100 \mathrm{~cm}$. The cutoff has a radius of $r_{c}=40$ cm where the density scale length is $L_{n} \equiv n /(d n / d r)=25 \mathrm{~cm}$.

Figure 2 displays the contour plot of the backward field amplitude $\left|E_{b}\right|$ for different values of the angular aperture of the probing beam $\left(\square_{0}\right)$. The field of turbulent fluctuations is the same in all four cases, with $\square_{n} \equiv<\tilde{n}^{2} / n^{2}>^{1 / 2}=1.0 \times 10^{-2}, \square_{r}=1.0 \mathrm{~cm}^{-1}$ and $q_{0}=40\left(\square_{p}=1.0 \mathrm{~cm}^{-1}\right)$. Figure 3 displays similar plots for a constant beam aperture $\left(\square_{0}=40^{\circ}\right)$ but different values of $\square_{n}$. In all four cases, the fluctuations have the same value of $q_{0}=20\left(\square_{p}=0.5 \mathrm{~cm}^{-1}\right)$ and $\square_{r}=1.0 \mathrm{~cm}^{-1}$, and identical sets of random phases $\left(\square_{p q}\right)$.

The plots of Figs. 2 and 3 illustrate how the radiation pattern of the backward field splits into several striations that seem to originate from a location behind the cutoff - a virtual cutoff - where the fluctuations in the backward field amplitude are at a their minimum. As noted in the previous section, the backward field does not coincide with the reflected wave in the plasma region. It is simply how the reflected wave, after crossing the region between the cutoff and the plasma edge, would appear to an observer in free space. Since the refractive index of this region is lower than one, the striations seem to start from a radial location $\left(r_{G}\right)$ behind the cutoff, at a distance from the latter of the order of the permittivity scale length, i.e., where the rays of waves originating near the real cutoff seem to intersect when observed in free space $[8,9]$. This phenomenon is similar to 
what makes the bottom of a swimming pool to appear artificially close to the surface, the only difference in this case being a refractive index larger than one.

As described in the introduction, interference of the spectral components of the reflected wave may result in a chaotic wave pattern in free space - the only place where we can perform reflectometry measurements. This is demonstrated in Fig. 4(a), which shows that the amplitude of the backward field at $r=r_{0}$ is strongly modulated by the fluctuations of Fig. 3(c). Furthermore, the fluctuating component ( $\tilde{\square}$ ) of the phase of $\tilde{n} / n$ (i.e., the change in the phase of the backward field due to the presence of fluctuations) is completely different from the phase of geometric optics $\tilde{\square}_{G O}(\square)=k_{0} \square_{c}^{b} b / \sqrt{[b} d r$ (Fig. $5(a))$. On the contrary, plasma fluctuations have a small effect on the value of $\left|E_{b}\right|$ near the virtual cutoff (Fig. 4(b)), where $\tilde{\square}$ coincides with $\tilde{\square}_{G O}$ (Fig. 5(b)). Consequently, since most of the contribution to $\tilde{\square}_{G O}$ comes from a narrow region in front of the cutoff, the poloidal power spectrum of $\tilde{\square}$ at $r=r_{G}$ must be similar to that of $\tilde{n} / n$ at $r=r_{c}$. This is indeed the case, as demonstrated in Fig. 6 where the spectrum of $\tilde{n} / n$ is normalized by the factor $\square_{r /} / k_{0}^{2} L_{n}$ (derived from the approximation of geometric optics $[5,6]$ ). Similarly to the results of previous simulations [9], we find that the best agreement is obtained using the value of $\tilde{n} / n$ at a small distance from the cutoff $(\sim 0.5 \mathrm{~cm}$ in Fig. 6$)$.

Apart from a few rare cases, such as that of Ref. [5], a normal procedure of standard reflectometry is to identify the spectrum of plasma turbulence with the spectrum of measured signals. This leads to erroneous results, as demonstrated by Fig. 7 showing the power spectra of $E_{b}$ at $r=r_{0}$ and of $\tilde{n} / n$ at $r=r_{c}$ (the latter being renormalized for facilitating the comparison with the backward field spectra). These results show very clearly that, as plasma fluctuations rise to the level found in tokamaks, the spectrum of reflected waves in free space - the main product of standard reflectometry - becomes considerably broader than the spectrum of fluctuations. 
As in previous simulations $[8,9]$, we find that the possibility of inferring the spectrum of plasma turbulence from the phase of the backward field breaks down at large levels of plasma fluctuations. This is explained by the fact that, since each spectral component of the backward wave originates near the corresponding reflecting point, the breakdown occurs when the set of these points is distributed over a distance $\Gamma r$ that is comparable to the radial scale of plasma fluctuations $\left(\square_{r}^{1}\right)$. This occurs when $[8,9]$

$$
\square_{n}<\frac{1}{\square^{3 / 4} L_{n} \square_{p}} .
$$

When this condition is not satisfied, we expect large fluctuations in the value of $\left|E_{b}\right|$ at the virtual cutoff and a departure of the spectrum of $\tilde{\square}$ (at $r=r_{G}$ ) from that of $\tilde{n} / n$ (at $\left.r=r_{c}\right)$. This is indeed what Fig. 8 shows when the value of $\square_{n}$ is raised to $3.0 \times 10^{-2}$ for fluctuations similar to those of Fig. $6\left(\square_{p}=0.5 \mathrm{~cm}^{-1}\right.$ and $\left.L_{n}=25 \mathrm{~cm}\right)$, in agreement with Eq. (16) that gives $\square_{n}<3.4 \times 10^{-2}$. Another demonstration of the validity of this criterion is provided by Fig. 9, where for $\square_{p}=1.0 \mathrm{~cm}^{-1}$ the breakdown occurs for a value of $\square_{n}$ smaller than $2 \times 10^{-2}$, again in agreement with Eq. (16) that for this case gives $\square_{n}<$ $1.7 \times 10^{-2}$

Finally, it is worth noting that when Eq. (16) is not satisfied, such as in Figs. 8(b) and $9(b)$, the power spectrum of $\tilde{\square}$ acquires a $1 / m^{2}$ dependence. This is reminiscent of the $1 / f^{2}$-frequency dependence of the spectra of standard reflectometry $[1,6]$.

\section{CONCLUSION}

In conclusion, we have presented a numerical study of microwave reflectometry for the measurement of turbulent fluctuations in a tokamak-like plasma with a cylindrical equilibrium configuration. Similarly to what was found previously in plane-stratified configurations, our results indicate that the characteristics of plasma fluctuations cannot 
be uniquely determined from the reflected waves if these are allowed to propagate freely to the point of detection, as in standard reflectometry. Again, we find that if the amplitude of fluctuations is below a threshold that is set by the spectrum of poloidal wave numbers (Eq. (16)), the local characteristics of density fluctuations can be obtained from the phase of reflected waves when these are collected with a wide aperture antenna and an image of the cutoff is formed (taking into account plasma refraction) onto an array of phase sensitive detectors. A reflectometer apparatus for testing this conjecture has been constructed and is currently being commissioned on the TEXTOR tokamak $[12,13]$. 


\section{ACKNOWLEDGEMENTS}

The author wishes to thank T. Munsat for useful discussions and for help in the visualization of numerical results.

This work was supported by U.S. DOE Contract No. DE-AC02-76-CHO-3073.

\section{REFERENCES}

[1] E. Mazzucato, Rev. Sci. Instrum. 69, 2201 (1998).

[2] W. Horton, Rev. Mod. Phys. 71, 735 (1999).

[3] J. W. Connor and H. R. Wilson, Plasma Phys. Control. Fusion 36, 719 (1994).

[4] E. Mazzucato, Bull. Am. Phys. Soc. 20, 1241 (1975); PPPL MATT-1151 (1975).

[5] E. Mazzucato and R. Nazikian, Phys. Rev. Lett. 71, 1840 (1993).

[6] R. Nazikian and E. Mazzucato, Rev. Sci. Instrum. 66, 392 (1995).

[7] W. B. Davenport and W. L. Root, Random signals and noise (McGraw-Hill, New York, 1958).

[8] E. Mazzucato, Rev. Sci. Instrum. 69, 1691 (1998).

[9] E. Mazzucato, Nucl. Fusion 41, 203 (2001).

[10] E. Mazzucato, T. Munsat, H. Park, B. H. Deng, C. W. Domier, and N. C.

Luhmann, Jr, A. J. H. Donné, and M. J. van de Pol, Phys. Plasmas 9, 1955 (2003)

[11] M. Abramowitz and I. A. Stegun, Handbook of mathematical functions (Dover, New York, 1965).

[12] T. Munsat, E. Mazzucato, H. Park, B. H. Deng, C. W. Domier, and N. C.

Luhmann, Jr, J. Wang, Z. G. Xia, A. J. H. Donné, and M. J van de Pol, Rev. Sci. Instrum. 74, 1426 (2003).

[13] T. Munsat, E. Mazzucato, H. Park, C.W. Domier, and N.C. Luhmann, A.J.H.

Donné, and M.J van de Pol, Plasma Phys. Control. Fusion 45, 469 (2003). 


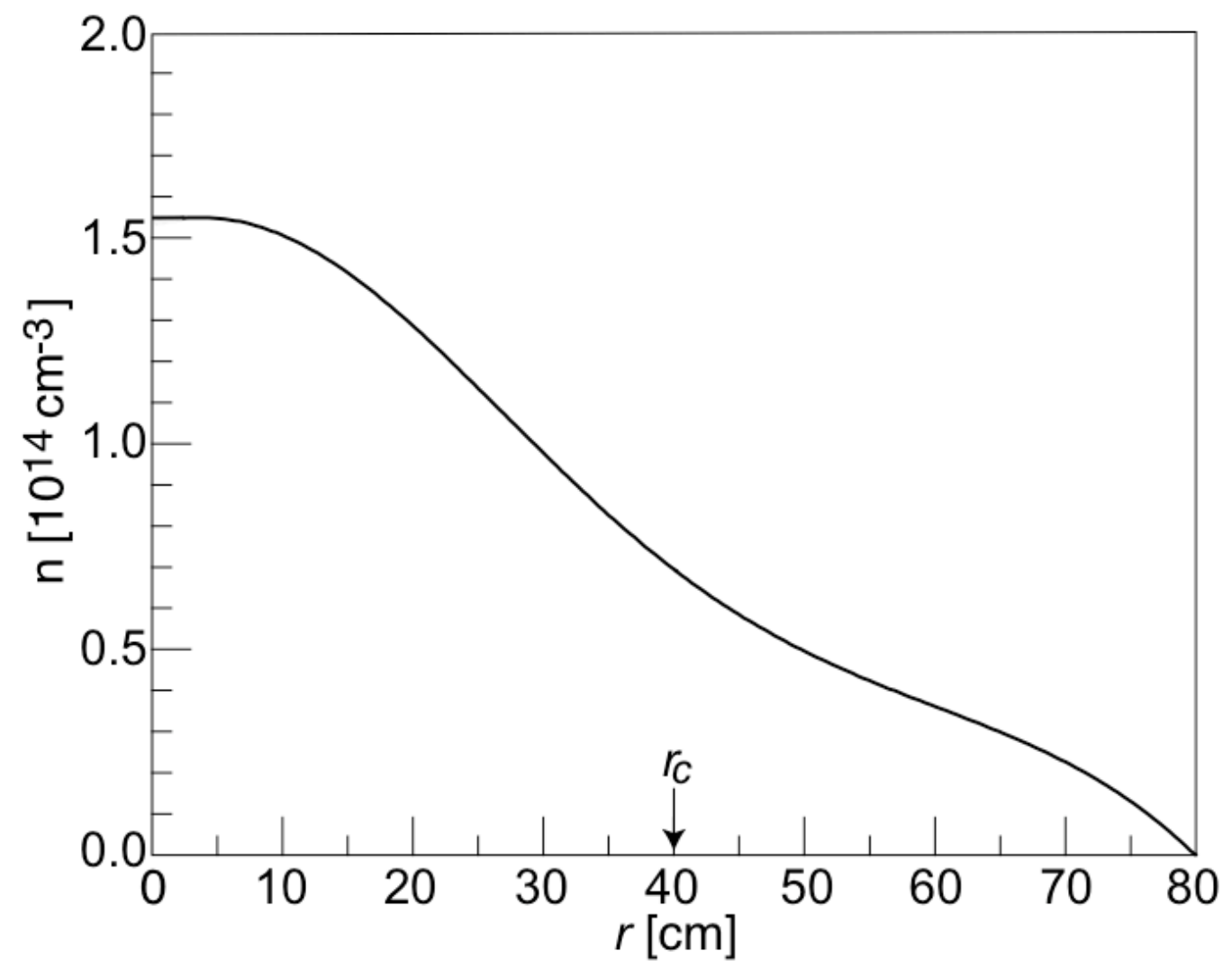

Figure 1. Plasma density profile; the cutoff is at $r=r_{c}$ for a probing wave with a frequency of $75 \mathrm{GHz}$ and the ordinary mode of propagation. 

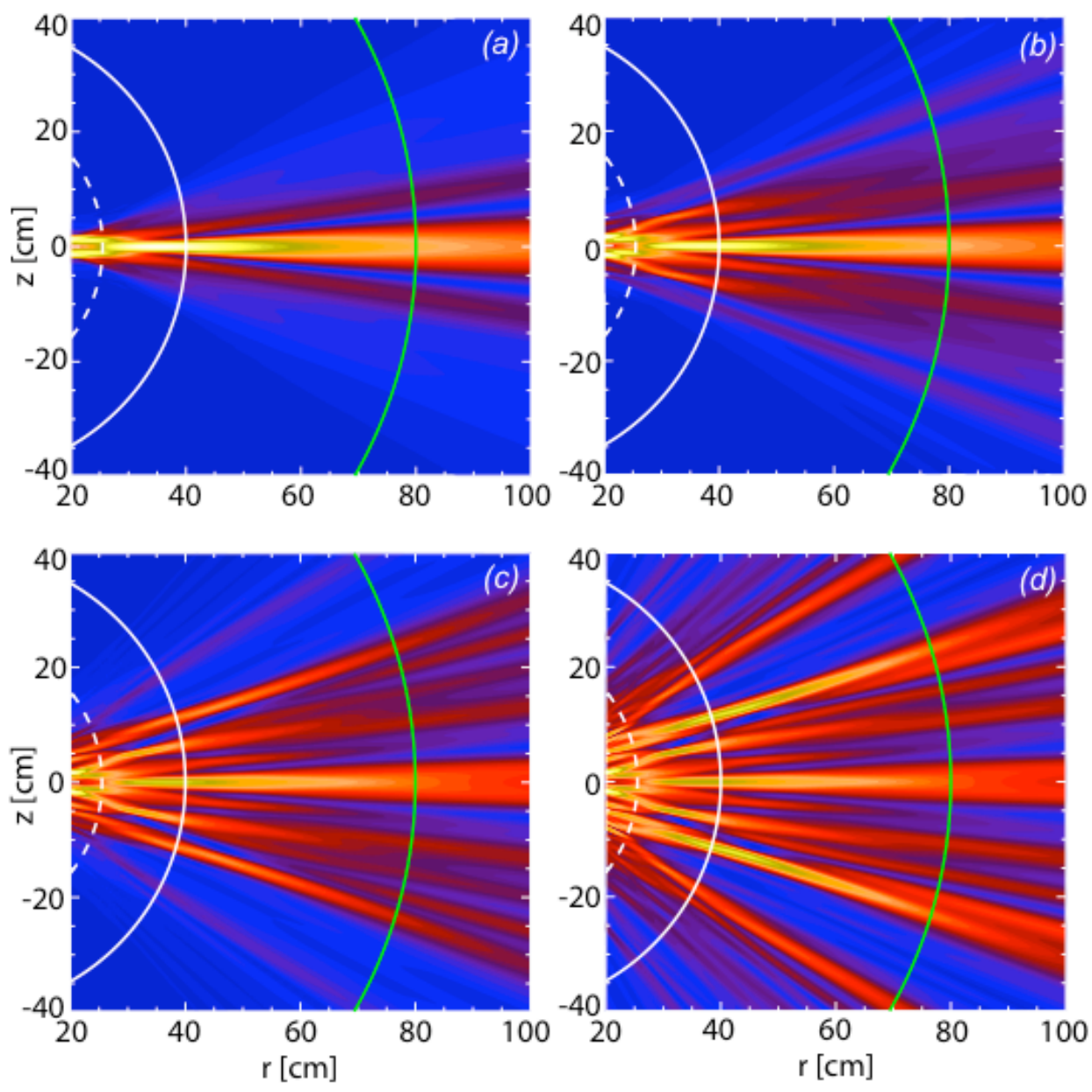

Figure 2. Contour plots of $\left|E_{b}\right|$ for different values of the angular width $\square_{0}: 5^{\mathrm{O}}(a), 10^{\mathrm{O}}$ $(b), 20^{\circ}(c), 40^{\circ}(d)$. Green line is the plasma boundary $\left(r_{b}=80 \mathrm{~cm}\right)$, solid white line is the cutoff $\left(r_{c}=40 \mathrm{~cm}\right)$, dashed line is the virtual cutoff $\left(r_{G}=26 \mathrm{~cm}\right)$. Fluctuations parameters: $q_{0}=40, \square_{r}=1.0 \mathrm{~cm}^{-1}$ and $\square_{n}=1.0 \times 10^{-2}$. 

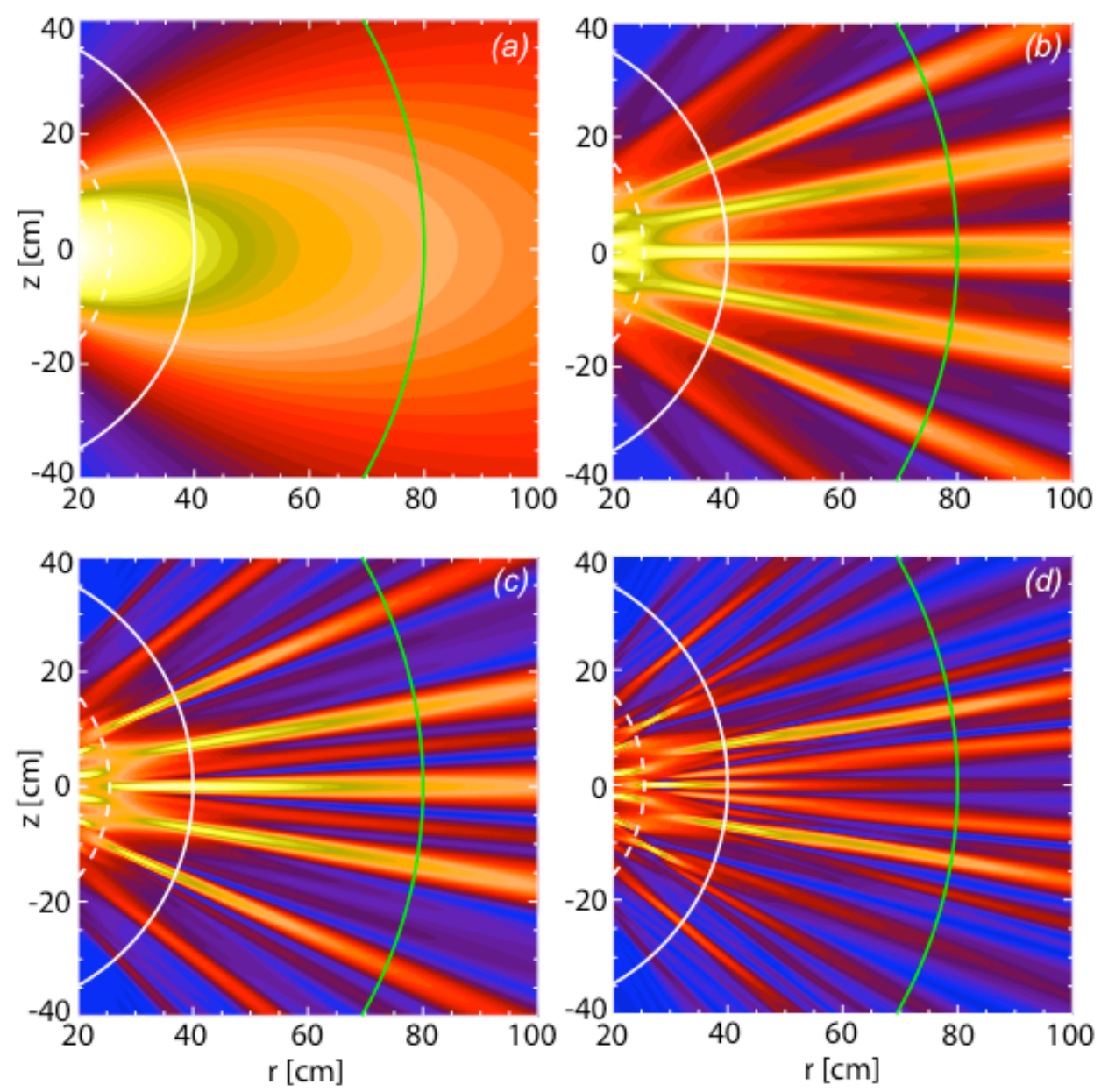

Figure 3. Contour plots of $\left|E_{b}\right|$ for different values of $\square_{n}: 0.0(a), 0.5 \times 10^{-2}(b), 1.0 \times 10^{-2}$ $(c), 2.0 \times 10^{-2}(d)$. Other parameters: $q_{0}=20, \square_{r}=1.0 \mathrm{~cm}^{-1}, \square_{0}=40^{\circ}$. 

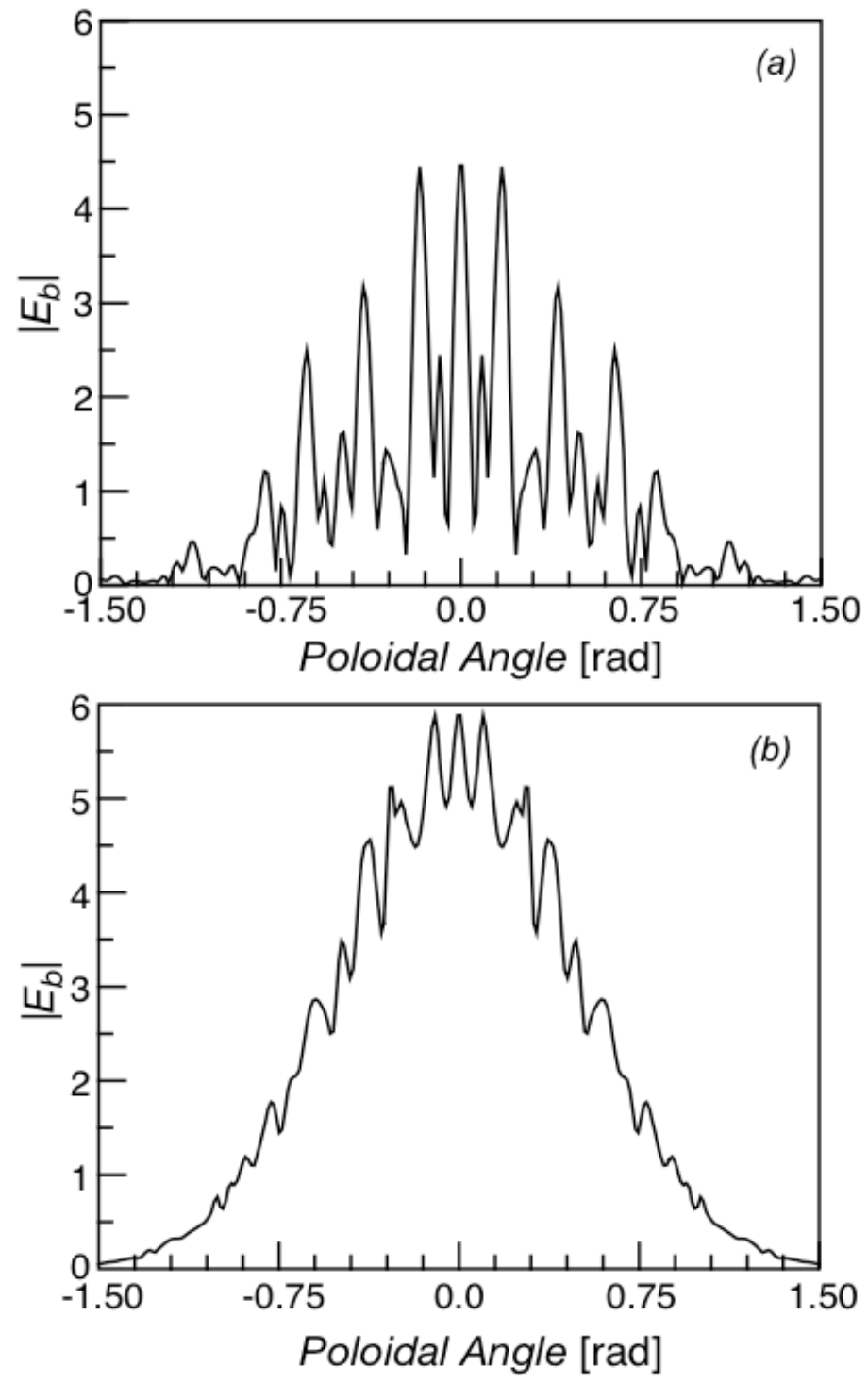

Figure 4. Profile of $\left|E_{b}\right|$ at $r=r_{0}(a)$ and $r=r_{G}(b)$ for the case of Fig. 3(c). 

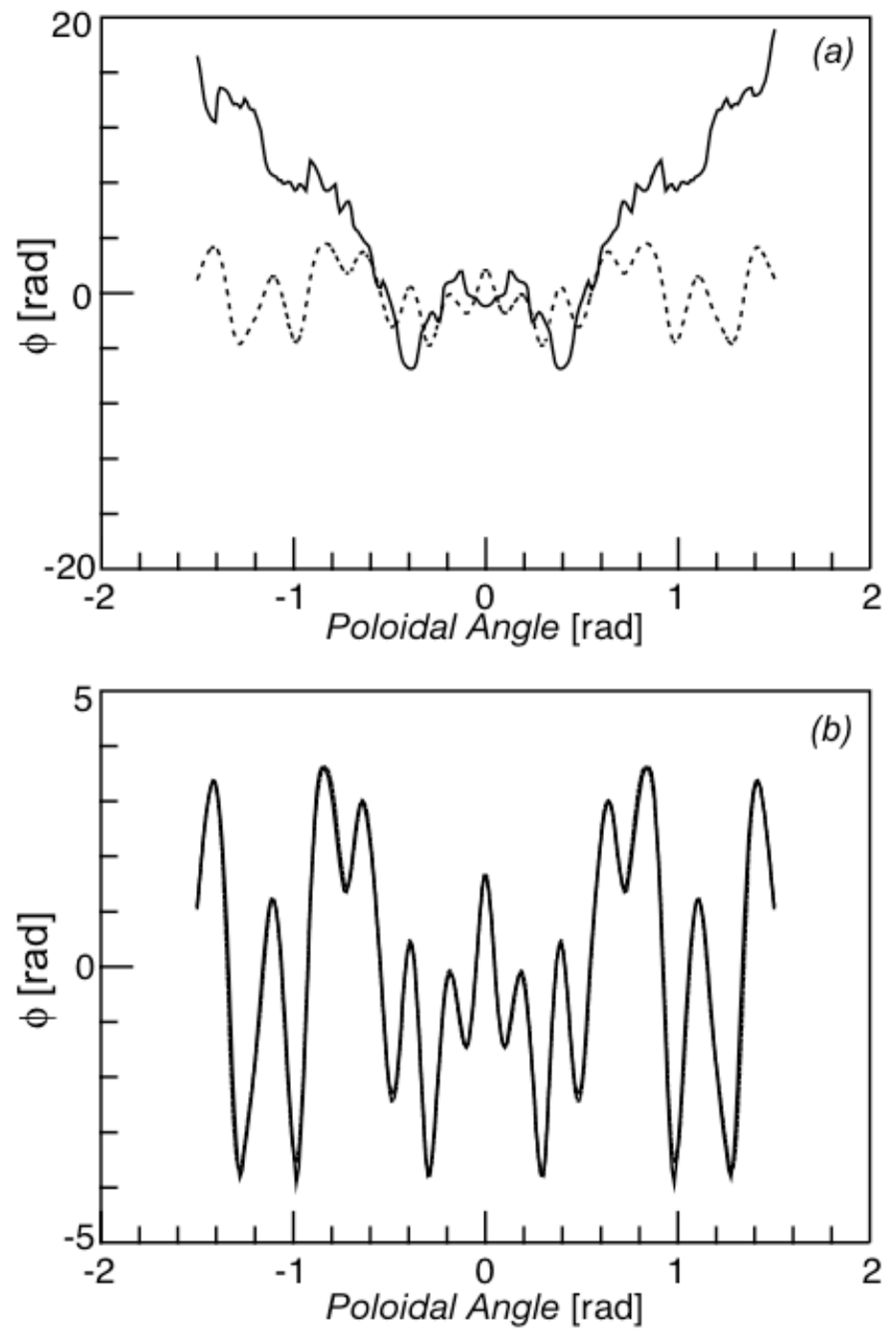

Figure 5. Comparison of $\tilde{\square}$ (solid line) and $\tilde{D}_{G O}$ (dashed line) at $r=r_{0}(a)$ and $r=r_{G}(b)$ for the case of Fig. 3(c). Note that the two curves in the bottom figure are almost identical. 

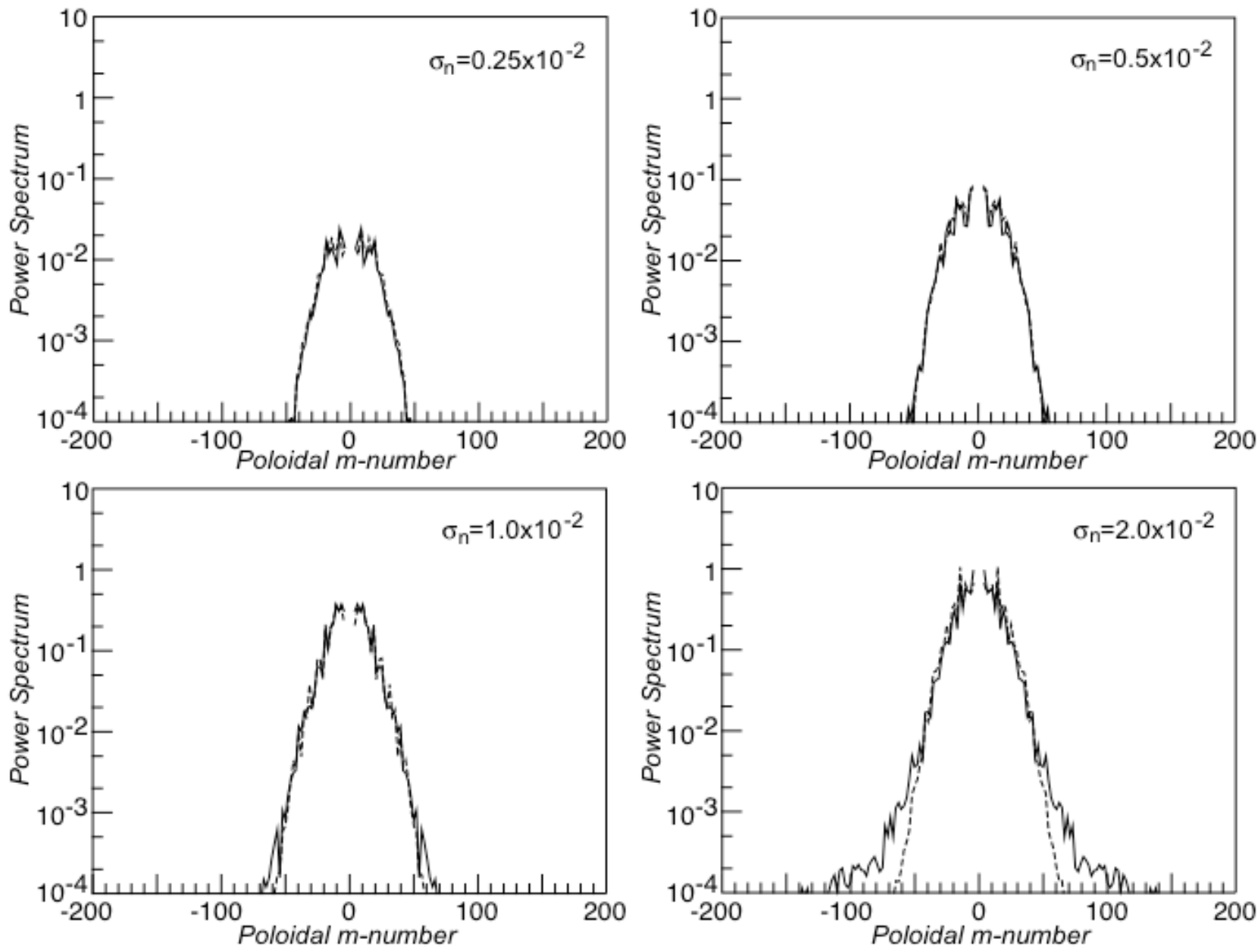

Figure 6. Power spectra of $\tilde{\square}$ at $r=r_{G}$ (solid lines) and of $\tilde{n} / n$ at $r \square r_{c}$ (dashed line) for different values of $\square_{n}$. Other parameters are those of Fig. 3. Spectra are averaged over twenty realizations of the turbulence field. 

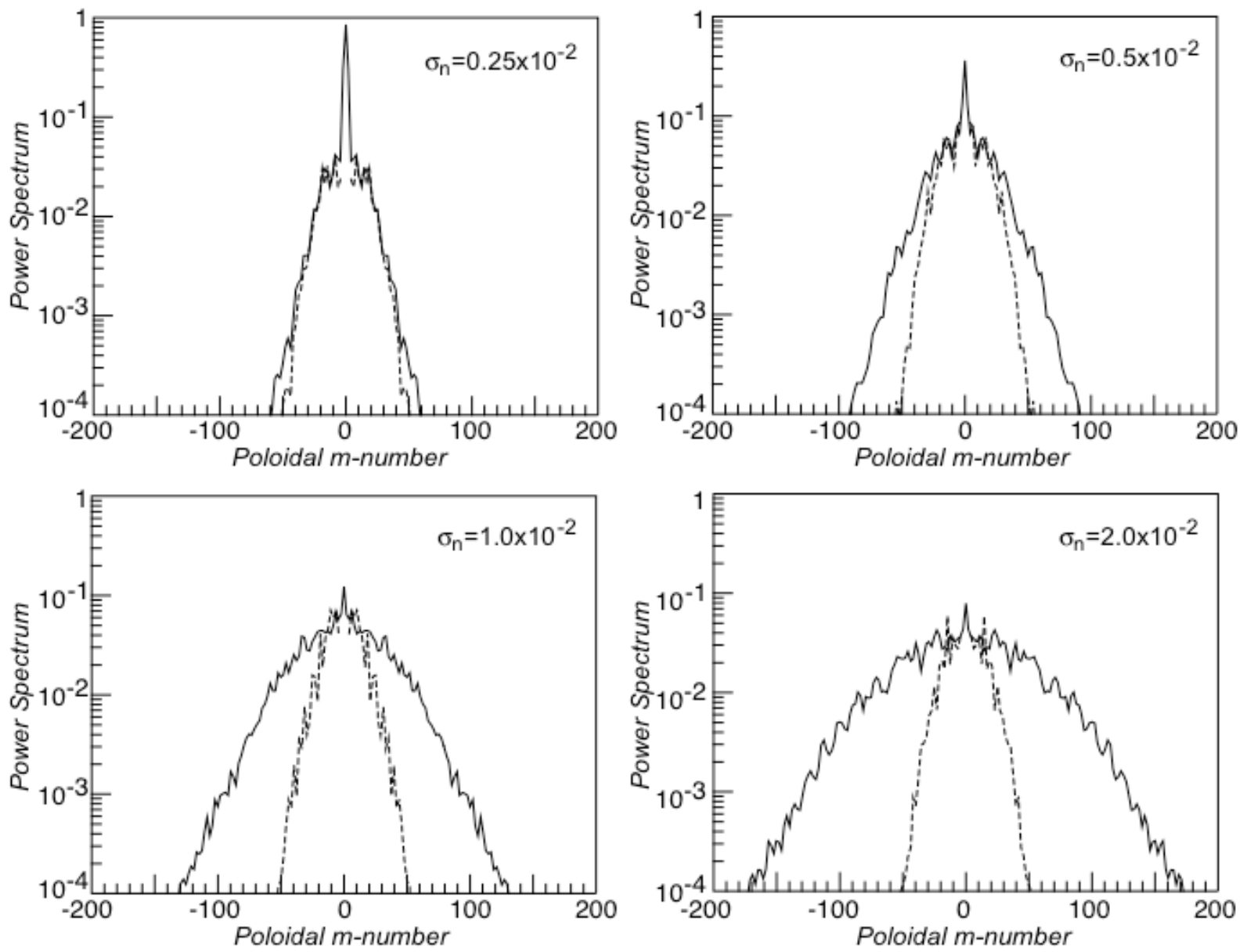

Figure 7. Power spectra of $E_{b}$ at $r=r_{0}$ (solid lines) and of $\tilde{n} / n$ at $r \square r_{c}$ (dashed line) for the fluctuations of Fig. 6. Spectra are averaged over twenty realizations of the turbulence field (density spectra are renormalized). 

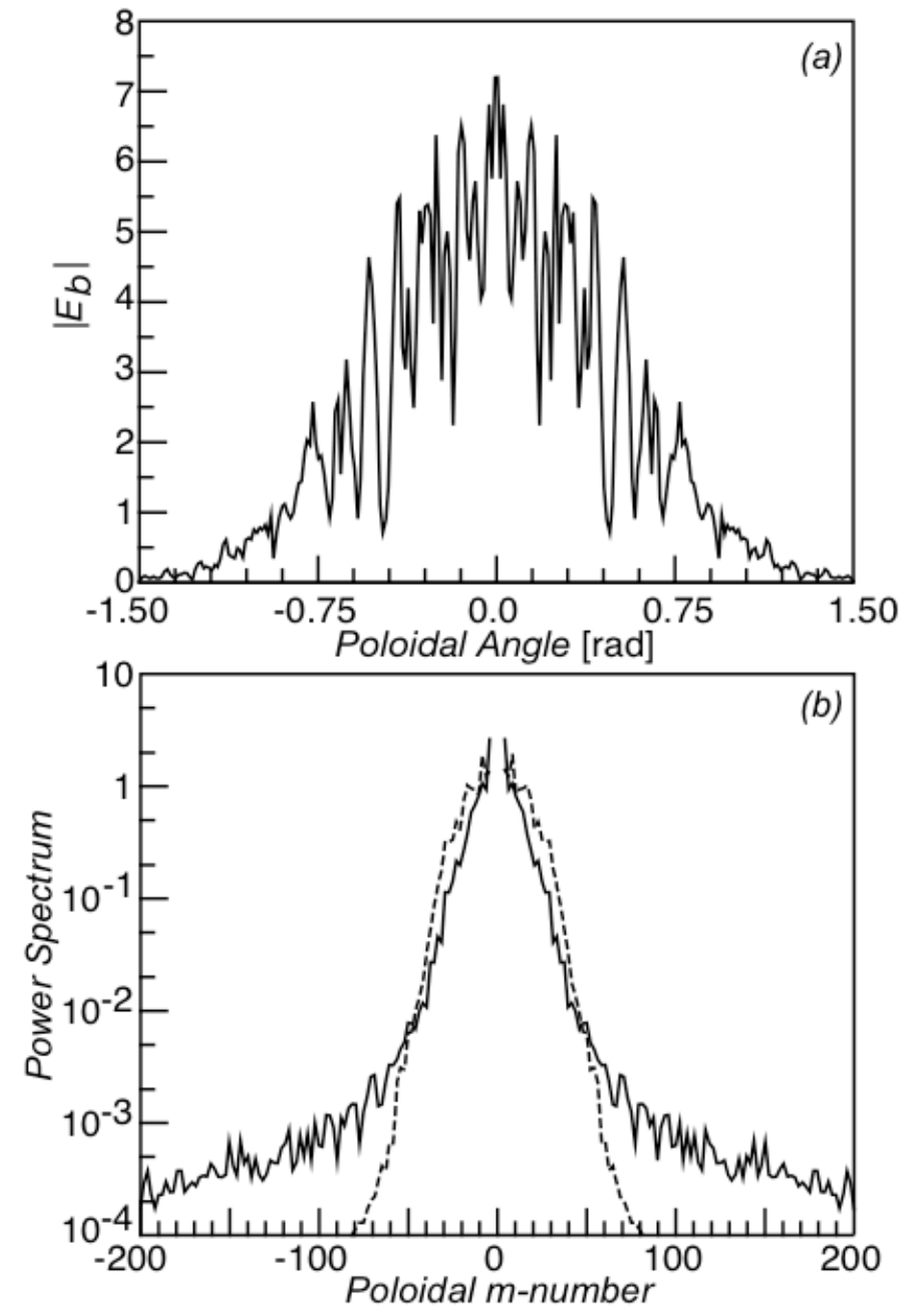

Figure 8. (a) Profile of $\left|E_{b}\right|$ at $r=r_{G}$. (b) Poloidal power spectrum of $\tilde{\square}$ at $r=r_{G}$ (solid line) and of $\tilde{n} / n$ at $r \square r_{c}$ (dashed line). Parameters are those of Fig. 3 with $\square_{n}=3.0 \times 10^{-2}$. Spectra are averaged over twenty realizations of the turbulence field. 

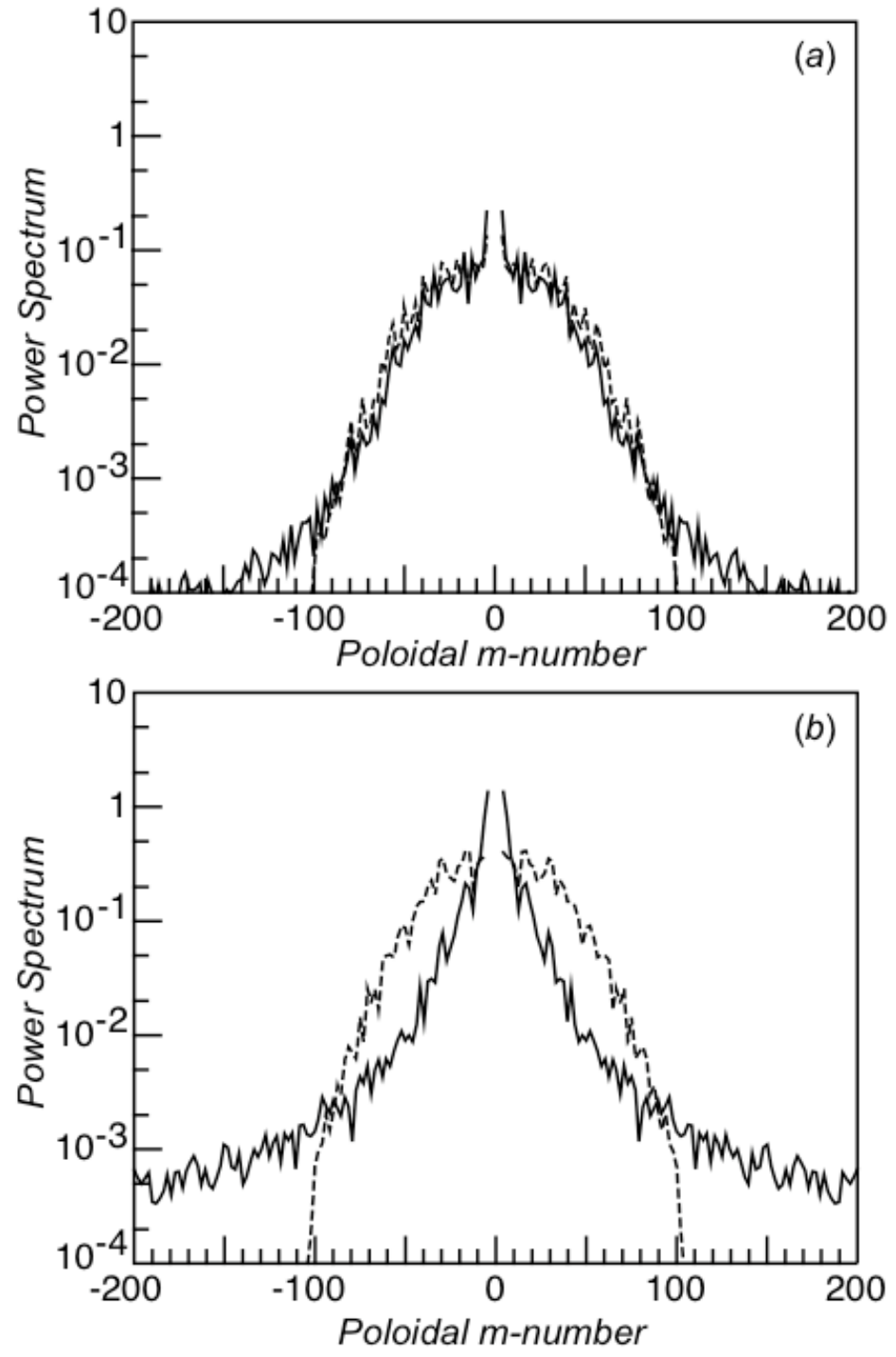

Figure 9. Same as in Fig. 8(b) for $q_{0}=40, \square_{r}=1.0 \mathrm{~cm}^{-1}, \square_{n}=1.0 \times 10^{-2}(a)$ and $\square_{n}=2.0 \times 10^{-2}(b)$. Spectra are averaged over twenty realizations of the turbulence field. 


\section{External Distribution}

Plasma Research Laboratory, Australian National University, Australia

Professor I.R. Jones, Flinders University, Australia

Professor João Canalle, Instituto de Fisica DEQ/IF - UERJ, Brazil

Mr. Gerson O. Ludwig, Instituto Nacional de Pesquisas, Brazil

Dr. P.H. Sakanaka, Instituto Fisica, Brazil

The Librarian, Culham Laboratory, England

Mrs. S.A. Hutchinson, JET Library, England

Professor M.N. Bussac, Ecole Polytechnique, France

Librarian, Max-Planck-Institut für Plasmaphysik, Germany

Jolan Moldvai, Reports Library, Hungarian Academy of Sciences, Central Research Institute for Physics, Hungary

Dr. P. Kaw, Institute for Plasma Research, India

Ms. P.J. Pathak, Librarian, Institute for Plasma Research, India

Ms. Clelia De Palo, Associazione EURATOM-ENEA, Italy

Dr. G. Grosso, Instituto di Fisica del Plasma, Italy

Librarian, Naka Fusion Research Establishment, JAERI, Japan

Library, Laboratory for Complex Energy Processes, Institute for Advanced Study, Kyoto University, Japan

Research Information Center, National Institute for Fusion Science, Japan

Dr. O. Mitarai, Kyushu Tokai University, Japan

Dr. Jiangang Li, Institute of Plasma Physics, Chinese Academy of Sciences, People's Republic of China

Professor Yuping Huo, School of Physical Science and Technology, People's Republic of China

Library, Academia Sinica, Institute of Plasma Physics, People's Republic of China

Librarian, Institute of Physics, Chinese Academy of Sciences, People's Republic of China

Dr. S. Mirnov, TRINITI, Troitsk, Russian Federation, Russia

Dr. V.S. Strelkov, Kurchatov Institute, Russian Federation, Russia

Professor Peter Lukac, Katedra Fyziky Plazmy MFF UK, Mlynska dolina F-2, Komenskeho Univerzita, SK-842 15 Bratislava, Slovakia

Dr. G.S. Lee, Korea Basic Science Institute, South Korea

Institute for Plasma Research, University of Maryland, USA

Librarian, Fusion Energy Division, Oak Ridge National Laboratory, USA

Librarian, Institute of Fusion Studies, University of Texas, USA

Librarian, Magnetic Fusion Program, Lawrence Livermore National Laboratory, USA

Library, General Atomics, USA

Plasma Physics Group, Fusion Energy Research Program, University of California at San Diego, USA

Plasma Physics Library, Columbia University, USA

Alkesh Punjabi, Center for Fusion Research and Training, Hampton University, USA

Dr. W.M. Stacey, Fusion Research Center, Georgia Institute of Technology, USA

Dr. John Willis, U.S. Department of Energy, Office of Fusion Energy Sciences, USA

Mr. Paul H. Wright, Indianapolis, Indiana, USA 
The Princeton Plasma Physics Laboratory is operated by Princeton University under contract with the U.S. Department of Energy.

\author{
Information Services \\ Princeton Plasma Physics Laboratory \\ P.O. Box 451 \\ Princeton, NJ 08543
}

Phone: 609-243-2750

Fax: 609-243-2751

e-mail: pppl_info@pppl.gov

Internet Address: http://www.pppl.gov 